\title{
OFFICERS OF THE ASSOCIATION
}

1924 Temporary Chairman-V. V. Anderson, M.D.

Temporary Secretary-Karl A. Menninger, M.D.

\section{President}

1924-26 William Healy, M.D.

*1926-27 Herman M. Adler, M.D.

1927-28 Karl A. Menninger, M.D.

1928-30 Lawson G. Lowrey, M.D.

1930-31 David M. Levy, M.D.

1931-32 Augusta F. Bronner, Ph.D.

*1932-33 Ira S. Wile, M.D.

1933-34 Frederick H. Allen, M.D.

1934-35 George S. Stevenson, M.D.

1935-36 Ralph P. Truitt, M.D.

1936-37 Edgar A. Doll, Ph.D.

1937-38 George J. Mohr, M.D.

1938-39 Frank J. O’Brien, M.D.

1939-40 Samuel W. Hartwell, M.D.

1940-41 Paul L. Schroeder, M.D.

*1941-42 Jacob Kasanin, M.D.

1942-43 Henry C. Schumacher, M.D.

1943-44 George H. Preston, M.D.

1944-46 Norvelle C. LaMar, M.D.

1946-47 Spafford Ackerly, M.D.

1947-48 Milton E. Kirkpatrick, M.D.

1948-49 S. J. Beck, Ph.D.

\section{Vice President}

*1924-26 Arnold Jacoby, M.D.

1926-27 George H. Reeve, M.D.

*1927-28 Ira S. Wile, M.D.

1928-30 Augusta F. Bronner, Ph.D.

1930-31 Edgar A. Doll, Ph.D.

1931-32 Ralph P. Truitt, M.D.

1932-33 Frederick H. Allen, M.D.

1933-34 Bertha C. Reynolds

1934-35 H. Meltzer, Ph.D.

1935-36 Willard C. Olson, Ph.D.

1936-37 Henry C. Schumacher, M.D.

1937-38 Elma Olson

1938-39 Almena Dawley

1939-40 E, K. Wickman

1940-41 Helen L. Witmer, Ph.D.

1941-42 Carl R. Rogers, Ph.D.

1942-43 Simon H. Tulchin

1943-44 S. J. Beck, Ph.D.

1944-46 Lawrence K. Frank

1946-47 Phyllis Blanchard, Ph.D.

1947-48 Marian McBee

1948-49 Edith B. Jackson, M.D.

\section{Secretary-Treasurer}

1924-26 Karl A. Menninger, M.D. *1926-27 Arnold Jacoby, M.D.

- Deceased
1927 Karl A. Menninger, M.D. Acting

1927-34 George S. Stevenson, M.D.

1934-35 Mary Augusta Clark

1935-37 George S. Stevenson, M.D.

1943-44 Milton E. Kirkpatrick, M.D.

\section{Treasurer}

1937-40 George S. Stevenson, M.D.

1940-43 Milton E. Kirkpatrick, M.D.

1944-49 James M. Cunningham, M.D.

Secretary

1937-43 Norvelle C. LaMar, M.D.

1944-48 Nina Ridenour, Ph.D.

1948-49 S. Harcourt Peppard, M.D.

\section{Councilors}

1924-26 V. V. Anderson, M.D.

1926-28 William Healy, M.D.

1928-30 Karl A. Menninger, M.D.

1930-31 Augusta F. Bronner, Ph.D.

1931-32 David M. Levy, M.D.

1932-33 Augusta F. Bronner, Ph.D.

1932-33 Ralph P. Truitt, M.D.

*1932-34 Herman M. Adler, M.D.

1933-35 George J. Mohr, M.D.

*1933-34 Ira S. Wile, M.D.

1934-35 Frederick H. Allen, M.D.

1934-36 Herbert E. Chamberlain, M.D.

1935-36 Mary Augusta Clark

1935-37 E. Van Norman Emery, M.D.

1936-38 Mildred C. Scoville

1936-37 Ralph P. Truitt, M.D.

1937-38 Edgar A. Doll, Ph.D.

1937-38 Harry M. Tiebout, M.D.

\section{Directors}

1938-39 Harry M. Tiebout, M.D. 1938-39 George J. Mohr, M.D.

1938-40 S. Spafford Ackerly, M.D.

1939-40 Frank J. O'Brien, M.D.

*1939-41 Jacob Kasanin, M.D.

1940-41 Samuel J. Hartwell, M.D.

1940-42 Simon H. Tulchin

1941-42 Paul L. Schroeder, M.D.

*1941-42 Everett Kimball, Ph.D.

*1942-43 Jacob Kasanin, M.D.

1942-43 Carmelite Janvier

1942-44 Katharine M. Wickman

1943-44 Henry C. Schumacher, M.D.

1943-44 Claudia Wannamaker

1943-46 H. S. Lippman, M.D.

1944-46 George H. Preston, M.D.

1944-47 Marian McBee

1946-47 Norvelle C. LaMar, M.D.

1946-48 S. Harcourt Peppard, M.D. 
1947-48 Spafford Ackerly, M.D.

1947-48 George E. Gardner, M.D.

1948-49 H. Whitman Newell, M.D.

1948-49 Milton E. Kirkpatrick, M.D.

1948-50 Harold H. Anderson, Ph,D.

\section{Editor}

*1924-26 Herman M. Adler, M.D.

1926-27 Karl A. Menninger, M.D.

*1927-30 Herman M. Adler, M.D.
1930-48 Lawson G. Lowrey, M.D.

1948-51 George E. Gardner, M.D.

President-Elect

1941-42 Henry C. Schumacher, M.D.

1942-43 George H. Preston, M.D.

1943-44 Norvelle C. LaMar, M.D.

1944-46 S. Spafford Ackerly, M.D.

1946-47 Milton E. Kirkpatrick, M.D.

1947-48 S. J. Beck, Ph.D.

1948-49 Marian McBee

MEETING PLACES OF THE A.O.A.

Organization Meeting, January 13, 1924

Annual Meetings

\begin{tabular}{|c|c|c|c|c|c|c|c|}
\hline 1 st & Chicago & June & 1924 & 13 th & Cleveland & February & 1936 \\
\hline $2 d$ & New York & May & 1925 & 14 th & New York & & 1937 \\
\hline $3 d$ & New York & June & 1926 & 15 th & Chicago & & 1938 \\
\hline 4 th & Cincinnati & June & 1927 & 16 th & New York & & 1939 \\
\hline 5 th & New York & February & 1928 & 17 th & Boston & & 1940 \\
\hline 6 th & New York & & 1929 & 18 th & New York & & 1941 \\
\hline 7 th & New York & & 1930 & 19th & Detroit & & 1942 \\
\hline 8 th & New York & & 1931 & 20 th & New York & & 1943 \\
\hline 9 th & Baltimore & & 1932 & $21 \mathrm{st}$ & Chicago & & 1944 \\
\hline 10 th & New York & & 1933 & $22 d$ & Cancelled & & 1945 \\
\hline 11 th & Chicago & & 1934 & $23 \mathrm{~d}$ & New York & & 1946 \\
\hline 12 th & New York & & 1935 & 24 th & Cincinnati & & 1947 \\
\hline & & & & 25 th & New York & April & 1948 \\
\hline
\end{tabular}

Twenty-Sixth Annual Meeting

Chicago, April 4, 5, 6, 1949 